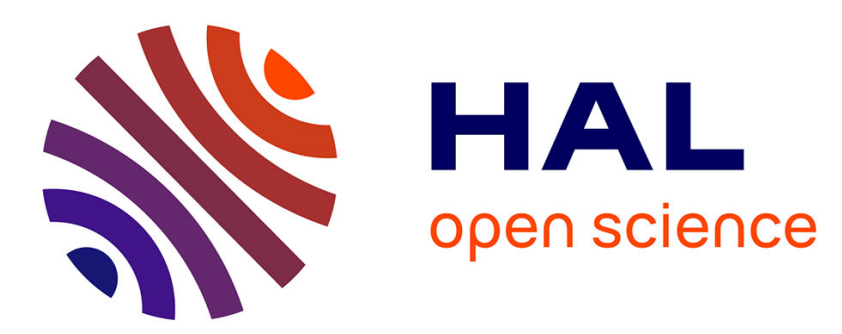

\title{
OPTICAL AND RADIATIVE PROPERTIES OF YSZ PLASMA-SPRAYED COATINGS
}

Vincent Debout, Franck Enguehard, E Meillot, Armelle Vardelle, Pierre Abelard, E Bruneton, Pierre Fauchais

\section{- To cite this version:}

Vincent Debout, Franck Enguehard, E Meillot, Armelle Vardelle, Pierre Abelard, et al.. OPTICAL AND RADIATIVE PROPERTIES OF YSZ PLASMA-SPRAYED COATINGS. 5th International Symposium on Radiative Transfer, Jun 2007, Bodrum, Turkey. 10.1615/ICHMT.2007.RadTransfProc.480 . hal-01287479

\section{HAL Id: hal-01287479 \\ https://hal.science/hal-01287479}

Submitted on 13 Mar 2016

HAL is a multi-disciplinary open access archive for the deposit and dissemination of scientific research documents, whether they are published or not. The documents may come from teaching and research institutions in France or abroad, or from public or private research centers.
L'archive ouverte pluridisciplinaire HAL, est destinée au dépôt et à la diffusion de documents scientifiques de niveau recherche, publiés ou non, émanant des établissements d'enseignement et de recherche français ou étrangers, des laboratoires publics ou privés. 


\title{
OPTICAL AND RADIATIVE PROPERTIES OF YSZ PLASMA-SPRAYED COATINGS
}

\author{
V. Debout , F. Enguehard ${ }^{* *}$, E. Meillot ${ }^{* *}$, A. Vardelle*, P. Abélard", E. Bruneton ${ }^{* *}$ and P. Fauchais \\ ${ }^{*}$ CNRS-SPCTS, University of Limoges, 123 avenue Albert Thomas, 87060 Limoges, France \\ *** CEA / Le Ripault, BP 16, 37260 Monts, France
}

\begin{abstract}
This paper is devoted to the study of the optical and radiative properties of YSZ plasma-sprayed coatings which are widely used as thermal barrier coatings (TBCs). The effects of microstructure and oxygen under-stoichiometry have been investigated by performing directionalhemispherical reflectance and transmittance measurements over the YSZ translucent spectral region of $0.25-8 \mu \mathrm{m}$. The effect of under-stoichiometry has been isolated by annealing samples in air at $500^{\circ} \mathrm{C}$ during various times. Using the Gouesbet-Maheu model, the radiative properties (absorption and scattering coefficients) of the coatings have been evaluated by numerical inversion of their optical spectra. The differences in the scattering coefficients are discussed in terms of pore size and volume fraction, while the absorption level is correlated to the under-stoichiometry of YSZ. A prediction of the radiative properties of the coatings based on their microstructural description is attempted by applying the Mie theory in an absorbing host medium (YSZ) including monosize spherical pores.
\end{abstract}

\section{INTRODUCTION}

YSZ plasma-sprayed coatings are widely used as thermal barrier coatings (TBCs) to protect metal parts from high temperature combustion gases in turbine engines [1]. To ensure high performance to TBCs, an extensive effort has been carried out to correlate the spraying parameters to the resulting microstructure and macrostrutural properties such as the thermal conductivity or the thermomechanical resistance. Hovewer, only a few studies $[1,2,3]$ have investigated the radiative properties of plasma-sprayed YSZ, although radiation becomes the major heat transfer mechanism at high temperature and the coating material (YSZ) is nearly translucent over the $0.5-5 \mu \mathrm{m}$ wavelength range where thermal radiation is mostly concentrated. Therefore, a better knowledge of the radiative properties of YSZ plasma-sprayed coatings is necessary to predict and optimize their insulating ability. A very difficult issue in such a study lies in the large variety of microstructural defects of plasmasprayed coatings and some possible chemical modifications of the material during plasma spraying such as oxygen reduction for a metal oxide in a reducing atmosphere [4,5]. Indeed, plasma-sprayed coatings are built-up on a prepared substrate by successive impinging, flattening and solidification of particles which were previously accelerated and heated in a plasma jet. The coatings exhibit a lamellar structure including a complex void structure with interlamellar pores, globular pores and intralamellar cracks. The size of the voids ranges from sub-micron to more than $10 \mu \mathrm{m}$ with various shapes (spherical shape for globular pores, flat shape for interlamellar pores and microcracks).

To our knowledge, no model is currently available to predict the radiative properties of such highly heterogeneous materials. To circumvent this difficulty, some simple phenomenological approaches are presented in this paper that aim to characterize the radiative properties of plasma-sprayed coatings and then to predict them from some simple microstructural descriptions. Our study is limited to the investigation of the optical and radiative properties of YSZ plasma-sprayed coatings at room temperature over the $0.5-8 \mu \mathrm{m}$ wavelength range where the microstructural effects are predominant. The effects of under-stoichiometry are also addressed by performing annealing in air at $500^{\circ} \mathrm{C}$ during 5 minutes to 24 hours and following the resulting variation of the optical and radiative properties.

This paper is organized as follows. In Section 1, our different experimental equipments are described. Section 2 is devoted to the results of the coating characterization that lead to classify our samples according to their main microstructural features such as the pore size, the pore shape and the lamellar 
structure (poor or high interlamellar contact). In Section 3, the optical properties of the coatings before and after annealing are presented, and the radiative properties (scattering and absorption coefficients) are deduced using an inversion method based on the Gouesbet-Maheu four flux model. Finally, Section 4 presents a tentative prediction of the radiative properties of YSZ plasma-sprayed coatings by applying the Mie theory to a host absorbing medium (YSZ) including monosize spherical pores.

\section{EXPERIMENTAL EQUIPMENTS}

\section{Materials and plasma spraying}

The sprayed powder is a commercial $\mathrm{ZrO}_{2}-8 \%$ wt $\mathrm{Y}_{2} \mathrm{O}_{3}$ powder (Amperit 827.054 from $\mathrm{H}$. C. Starck) manufactured by spray drying, agglomeration and sintering with a particle size ranging from 22 to 45 $\mu \mathrm{m}$ after analysis. The substrate is a stainless steel (X13M grade from Aubert \& Duval, France) disk ( $25 \mathrm{~mm}$ in diameter and $5 \mathrm{~mm}$ in thickness) grit blasted to $\mathrm{Ra}=4 \mu \mathrm{m}$ before spraying.

Air plasma spraying was performed using a F4-VB torch (Sulzer Metco AG, Switzerland) with nozzles of different diameters. The powder was injected through a $1.5 \mathrm{~mm}$ internal diameter injector perpendicular to the torch axis. The powder carrier gas was adjusted in such a way that the median trajectory of the spray jet made an angle of $3.5^{\circ}$ with the torch axis. After a substrate preheating stage, the coating temperature was maintained at more than $200^{\circ} \mathrm{C}$ using a cryogenic argon system. It must be emphasized here that the cryogenic argon system leads to a decrease in oxygen content in the spraying atmosphere which consequently affects the oxygen stoichiometry of the coatings. Indeed, the YSZ particles first undergo an oxygen reduction in the plasma core stimulated by high temperatures $\left(8000\right.$ to $12000 \mathrm{~K}$ ), a long dwelling time and the plasma reducing gas $\left(\right.$ mainly $\mathrm{H}_{2}$ ) $[4,5]$. A subsequent oxydation of the particles can take place with the engulfment of the surrounding gas in the plasma plume, which produces a reduction of the oxygen deficit. Oxygen rarefaction (from $21 \%$ to $5 \% \mathrm{vol}$ for high temperature parameters) due to the cryogenic argon prevents this latter reaction and results in dark YSZ coatings.

The coating deposition operating conditions were selected in order to obtain a large range of particle velocities and melting states at the impact on the substrate by using two particle diagnostic tools: (1) the DPV-2000 system (Tecnar Automation, Quebec, Canada) based on the detection and analysis of particle radiation [6] that enables the determination of the particle velocity and temperature (no absolute value for this latter parameter as reported in [7]); (2) a particle collection device with two cryogenic argon nozzles that allows to quench particles at their melting state which is then studied by image analysis of scanning electronic microscopy (SEM) cross-sectional views of collected particles.

\section{Microstructural characterization tools}

Before characterization, the coatings were separated from their substrates by immersion in an aqua regia solution (mixture of hydrochloric acid and nitric acid) at room temperature during 1 to 2 hours. The open porosity network was characterized by Archimedes porosimetry and mercury intrusion porosimetry (MIP), this latter technique allowing also the determination of the pore size distribution between $100 \mu \mathrm{m}$ and about $10 \mathrm{~nm}$ by applying injection pressures ranging from $0.1 \mathrm{up}$ to $400 \mathrm{MPa}$. Further information about the microstructure were provided by SEM observations of prepared samples after a chemical etching with $\mathrm{HF}$ acid $(50 \% \mathrm{vol})$ for 5 to 10 minutes at room temperature. To obtain a quantitative description of the coating microstructure, a digital image analysis were performed. The magnification x 2000 was selected as a compromise between the visualization of thin details and the visualization of an area representative of the porous structure. The following features were analysed: the relative fraction of spherical pores (sphericity $>0.9$ ), cracks and intermediate shape and the relative distance between observable splat boundaries. 


\section{Optical characterization tools}

Room temperature directional-hemispherical reflectance and transmittance spectra of plasmasprayed coatings were collected over the $0.25-8 \mu \mathrm{m}$ spectral range for $500 \mu \mathrm{m}$ thick coatings before and after annealing at $500^{\circ} \mathrm{C}$ in air for 5 minutes to 24 hours. Two equipments were used: a Varian Cary 5000 grating spectrometer over the $0.25-2.5 \mu \mathrm{m}$ range equipped with a $110 \mathrm{~mm}$ diameter PTFE integrating sphere, and a mid-IR Brucker IF66v/s FTIR spectrometer mounted with a $76 \mathrm{~mm}$ diameter gold coated integrating sphere. To avoid, as much as possible, the absorption contribution of atmospheric $\mathrm{H}_{2} \mathrm{O}$ and $\mathrm{CO}_{2}$, the data were collected under soft vacuum (2000-3000 Pa). The spectra measured with the Brucker spectrometer were adjusted and merged with the Varian data between 1.5 and $2.5 \mu \mathrm{m}$ using the dedicated OPUS software (Bruker Optik $\mathrm{GmbH}$ ) in order to examine the spectra over the entire wavelength range.

\section{MICROSTRUCTURAL DESCRIPTION OF THE YSZ PLASMA-SPRAYED COATINGS}

The four selected spray conditions lead to a large range in particle velocity and melting state at the impact on the substrate (Table 1). Since these characteristics govern the particle viscosity and kinetic energy and thus control the flattening and solidification processes on the substrate, four coatings with significant microstructural differences were obtained (Fig. 1).

Table 1. Spraying conditions and particle characteristics at the impact on the substrate.

\begin{tabular}{cccccc}
\hline $\begin{array}{c}\text { Condition } \\
\text { set } \\
\text { number }\end{array}$ & $\begin{array}{c}\text { Plasma gas } \\
\text { volume flow } \\
\text { rates (slpm) } \\
\text { Ar/He/H } / \mathrm{H}_{2}\end{array}$ & $\begin{array}{c}\text { Total gas } \\
\text { mass flow } \\
\text { rate }(\mathrm{g} / \mathrm{s})\end{array}$ & $\begin{array}{c}\text { Specific } \\
\text { enthalpy } \\
(\mathrm{MJ} / \mathrm{kg})\end{array}$ & $\begin{array}{c}\text { Particle characteristics at the } \\
\text { impact on the substrate } \\
\text { Melting } \\
\text { state }\end{array}$ & $\begin{array}{c}\text { Mean velocity } \\
(\mathrm{m} / \mathrm{s})\end{array}$ \\
\hline 1 & $45 / 0 / 15$ & 1.359 & 19.5 & High & $280 \pm 60$ \\
\hline 2 & $65 / 30 / 5$ & 2.027 & 10.7 & Poor & $300 \pm 70$ \\
\hline 3 & $12 / 45 / 3$ & 0.495 & 24.0 & Average & $150 \pm 25$ \\
\hline 4 & $6 / 12 / 1.5$ & 0.226 & 14.2 & High & $50 \pm 10$ \\
\hline
\end{tabular}

Sets 1 and 2 of spray conditions lead to dense microstructures and lamellae that are difficult to discern even after the etching stage. This can be related to the high particle velocity causing an important deformation at the impact on the substrate and thus providing thin cohesive lamellae. Conversely, a low particle velocity leads to a poor flattening of the particles on the substrate for coating 4 . The lamellae are thicker and some particles remain undistorted after the impact. These are probably large particles with temperature slightly over the melting temperature of $\operatorname{YSZ}\left(2750^{\circ} \mathrm{C}\right)$. Coating 3 exhibits a chaotic microstructure with interconnected coarse pores and partially melted or even unmelted particles. This can be related, at least in part, to the intermediate velocity value and the average melting state of the impinging particles that reduce the flattening degree.

A large range of porosity (11-23\%) is obtained, with a pore size covering an order of magnitude from the dense coatings (sets 1 and 2) (pore mean size down to $0.16 \mu \mathrm{m}$ ) to coatings 3 and 4 (pore mean size up to $1.6 \mu \mathrm{m}$ ). The morphological analysis shows that the pore shape is essentially intermediate between spheres and cracks. Besides, it appears that coatings 1 and 2 exhibit a larger content of spherical pores. This result is somewhat illogical since a better flattening degree due to particle high velocity is usually consistent with a lower content in globular pores [8]. This highlights the limitations of our approach. For example, the distinction between cracks and other shapes of voids is quite difficult to define for wide cracks. Moreover, the problem of finding a magnification that is representative of the overall structure is rather tricky owing to the large size range of the defects. The investigation of the lamellar structures raises the same problem: coating 1 presents the highest distance 
between horizontal cracks although its lamellae are very thin. This can be ascribed to the difficulty to discern splat boundaries even after a chemical etching.
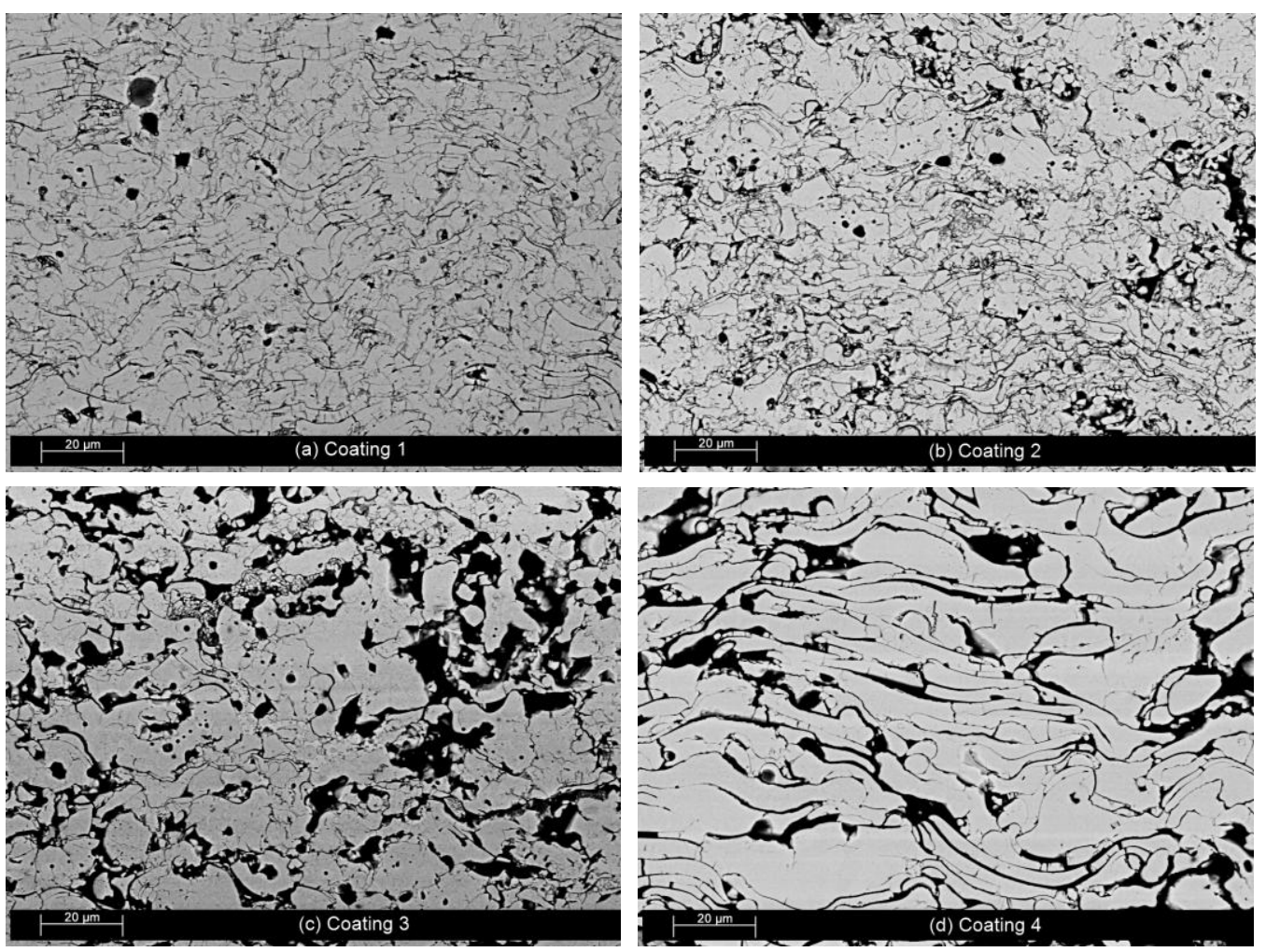

Figure 1. SEM cross-sectional observations of the four YSZ plasma-sprayed coatings.

Since no radiative model is able to integrate all the microstructural diversity of plasma-sprayed coatings, it seems better to classify the samples according to the pore size, the porosity content and these partial results of digital analysis. The microstructural classification that we have retained and the corresponding description for radiative modelling are reported in Table 2.

Table 2. Microstructural classification and simple description of our YSZ plasma-sprayed coatings.

\begin{tabular}{ccccc}
\hline $\begin{array}{c}\text { Class of plasma- } \\
\text { sprayed coatings }\end{array}$ & $\begin{array}{c}\text { Sample } \\
\text { number }\end{array}$ & $\begin{array}{c}\text { Porosity } \\
(\%)^{\mathrm{a}}\end{array}$ & $\begin{array}{c}\text { Pore mean } \\
\text { size }(\mu \mathrm{m})^{\mathrm{b}}\end{array}$ & Simple description \\
\hline $\begin{array}{c}\text { Dense coating with a } \\
\text { thin lamellar structure }\end{array}$ & $1-2$ & $11-12$ & $0.25-0.16$ & $\begin{array}{c}\text { Homogeneous matrix with } \\
\text { spherical submicron-sized pores }\end{array}$ \\
\hline $\begin{array}{c}\text { Porous coating with a } \\
\text { lamellar structure }\end{array}$ & 4 & 18 & 1.0 & $\begin{array}{c}\text { Multilayer medium consisting of } \\
\text { air and YSZ slabs }\end{array}$ \\
\hline $\begin{array}{c}\text { Porous coating with } \\
\text { coarse pores }\end{array}$ & 3 & 23 & 1.6 & $\begin{array}{c}\text { Homogeneous matrix with } \\
\text { randomly oriented spheroid pores }\end{array}$ \\
\hline
\end{tabular}

${ }^{a}$ Determined by Archimedes porosimetry. ${ }^{b}$ Determined by MIP.

\section{OPTICAL AND RADIATIVE CHARACTERIZATION OF THE COATINGS}

\section{Optical properties}

The directional-hemispherical reflectance and transmittance spectra of the four as-sprayed coatings (500 $\mu \mathrm{m}$ thick) over the 0.25-8 $\mu \mathrm{m}$ range are shown in Fig. 2. 

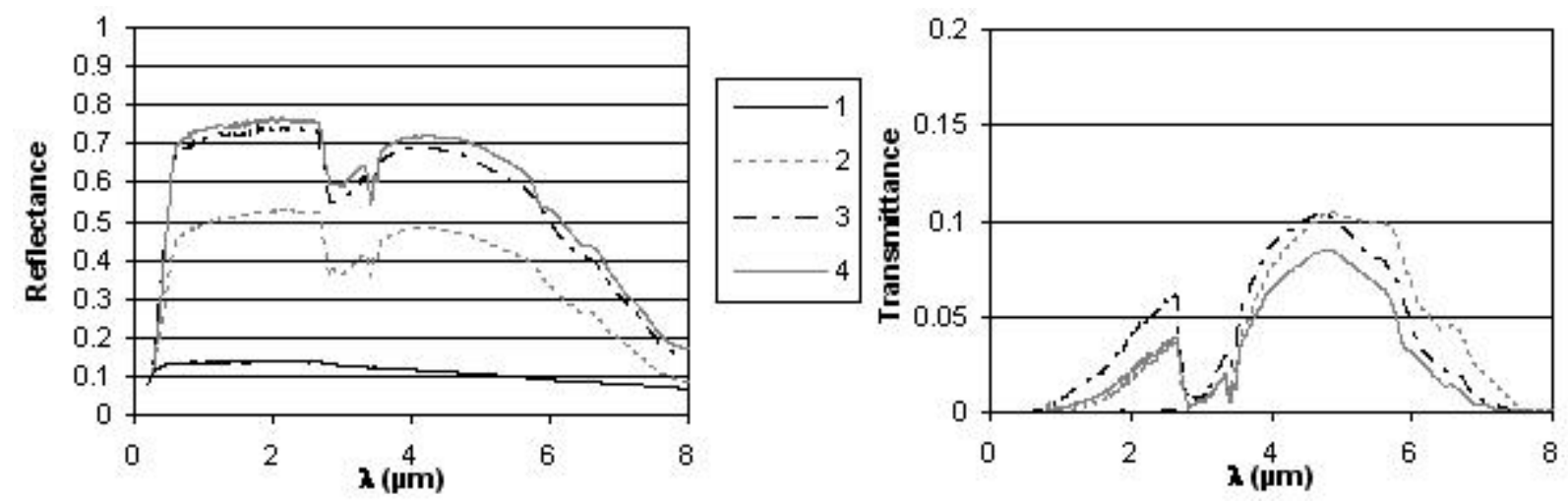

Figure 2. Reflectance and transmittance spectra of the four as-sprayed coatings.

While the YSZ material is translucent over the $0.5-5 \mu \mathrm{m}$ band and becomes semi-transparent up to 8 $\mu \mathrm{m}$ due to an increase in intrinsic absorption (multiphonon process), the four YSZ as-sprayed coatings exhibit different optical behaviours. First, contrary to dense YSZ, a decrease in transmittance and reflectance is noticed over the 2.5-4 $\mu \mathrm{m}$ range. This large absorption band is due to the $\mathrm{O}-\mathrm{H}$ bond and the presence of free water within the porosity of the YSZ plasma-sprayed samples as already described in [2]. A more striking point is the zero transmittance of dense coating 1 and its weak reflectance as compared to porous samples such as coatings 3 and 4. This significant difference in optical properties can be related to the sample colour, $i$. e. to the YSZ under-stoichiometry during spraying [5]. This phenomenon is enhanced by a high hydrogen content such as in condition 1 (Table 1) that promotes a good heat treatment to the particles and consequently requires more cryogenic argon flow to maintain the substrate temperature at $200^{\circ} \mathrm{C}$. Therefore, the four selected spray conditions result in coatings with various colors: dark for coating 1, pale gray for coating 2 and white for coatings 3 and 4 .

Annealing and optical properties. To reduce the oxygen deficit in the YSZ plasma-sprayed coatings, an annealing procedure in air at $500^{\circ} \mathrm{C}$ has been carried out for 5 minutes to 24 hours. Since no significant microstructural modifications have been observed after treatment, the variations in the optical spectra may reasonably be attributed to the evolution in oxygen stoichiometry within the YSZ material. The results obtained for coating 1 (initially dark) are presented in Fig. 3.
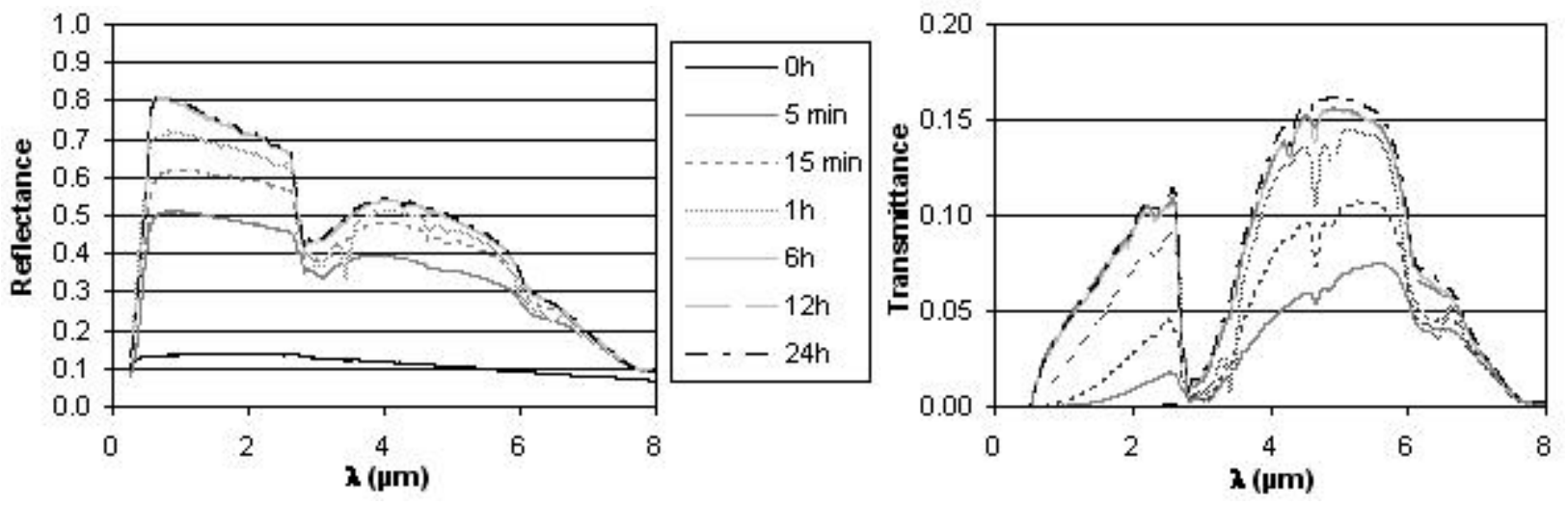

Figure 3. Effect of the annealing duration at $500^{\circ} \mathrm{C}$ on the optical spectra of coating 1.

By decreasing the oxygen deficit within the YSZ material, the annealing process leads to a strong increase in transmittance and reflectance. This evolution is marked even after a short annealing (5 minutes) and illustrates the strong optical dependence on the matrix stoichiometry. Beyond 12 hours, the optical properties do not vary any longer, and at that point the optical behaviour is a signature of the coating microstructure. 
Microstructural effects. The directional-hemispherical reflectance and transmittance of the four coatings after annealing at $500^{\circ} \mathrm{C}$ during 24 hours are reported in Fig. 4.
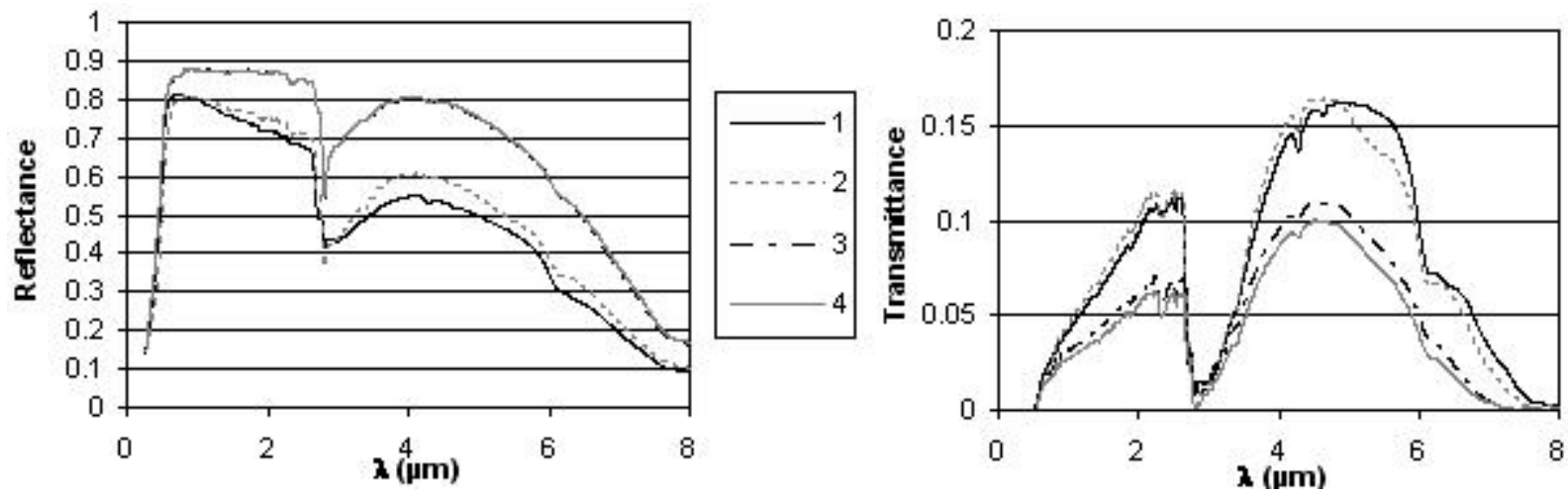

Figure 4. Optical spectra of the four coatings after annealing at $500^{\circ} \mathrm{C}$ for 24 hours.

At first glance, the reflectance and transmittance levels can be linked to both the size and the volume fraction of pores within the plasma-sprayed samples. Coatings 3 and 4 with high porosities $(\approx 20 \%)$ and micron-sized pores exhibit high reflectance and low transmittances, while coatings 1 and 2 with low porosities and submicron-sized pores display lower reflectance and higher transmittance. Such behaviours illustrate the strong scattering feature of plasma-sprayed coatings as shown in $[2,3]$.

\section{Radiative properties}

The absorption $\mathrm{k}$ and scattering $\mathrm{s}$ coefficient spectra were deduced by numerical inversion of the experimental reflectance and transmittance spectra of the four coatings using the Gouesbet-Maheu four-flux model. A detailed description of this theory is available in [9]. A preliminary optical homogenization of the coatings using the Bruggeman formalism was performed to provide their effective dielectric function spectra $\varepsilon_{\text {eff }}=\left(\mathrm{n}_{\text {eff }}-\mathrm{j} \kappa_{\text {eff }}\right)^{2}$.

For an accurate determination of the radiative properties, the boundary conditions (interface reflection) and the intrinsic parameters of the Gouesbet-Maheu model $\zeta$ and $\varepsilon$ have been evaluated cautiously. The reflection boundary conditions at the interfaces have been calculated with the assumption of negligible absorption $\left(\kappa_{\text {eff }} \approx 0\right)$ using the Fresnel relation for specular reflection and integrating it over a half-sphere for diffuse internal reflection. The first parameter $\zeta$ of the four-flux model is the forward scattering ratio $i$. e. the ratio of the forward scattered energy over the whole scattered energy. Based on this definition, $\zeta$ has been derived from the asymmetry factor $\mathrm{g}$ defined in the Mie theory and evaluated from the mean pore size (see formula reported in [10]). The second parameter $\varepsilon$ describes the increase in the light path length within the medium due to scattering. Vargas et al [11] have recently expressed it as a linear function of $\zeta: \varepsilon=3-2 \zeta$.

Annealing effects. Fig. 5 displays the evolution of the absorption and scattering coefficient spectra versus the annealing duration deduced from the experimental optical spectra of coating 1 (Fig. 3).

By increasing the annealing duration at $500^{\circ} \mathrm{C}$ in air, the oxygen deficit is gradually removed in the YSZ matrix, leading to a decrease in absorption. This decrease is significant even after a short annealing, the path length of light within the sample is then increased, more scatterers can then be struck by light, and the scattering phenomenon is enhanced. This result confirms the strong effect of oxygen under-stoichiometry on the optical and radiative properties of YSZ plasma-sprayed coatings. 

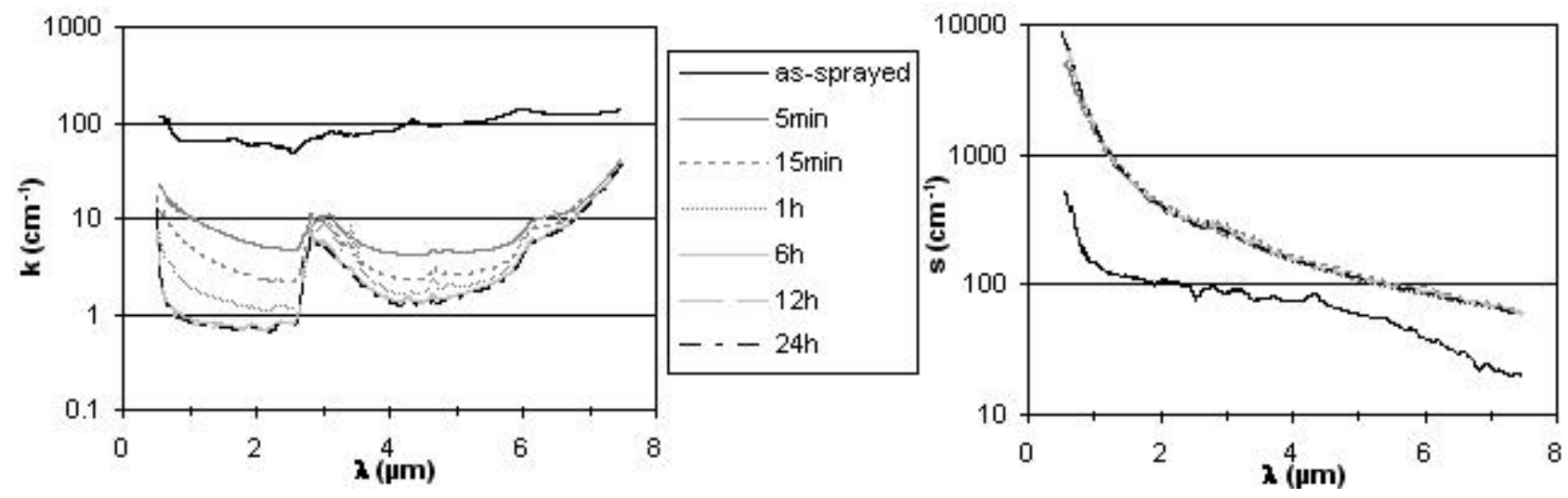

Figure 5. Effect of the annealing duration at $500^{\circ} \mathrm{C}$ on the radiative coefficient spectra of coating 1.

Microstructural effects. The absorption k and scattering s coefficient spectra of the four plasmasprayed coatings after a 24 hour annealing are reported in Fig. 6.

The right graph of Fig. 6 clearly shows two distinct radiative behaviours of the four samples: coatings 1 and 2 exhibit a scattering peak at short wavelengths $(<1.5 \mu \mathrm{m})$ and low scattering values beyond, whereas coatings 3 and 4 display high scattering values over a larger spectral band $(0.5-3 \mu \mathrm{m})$ while a slow decrease is observed beyond.
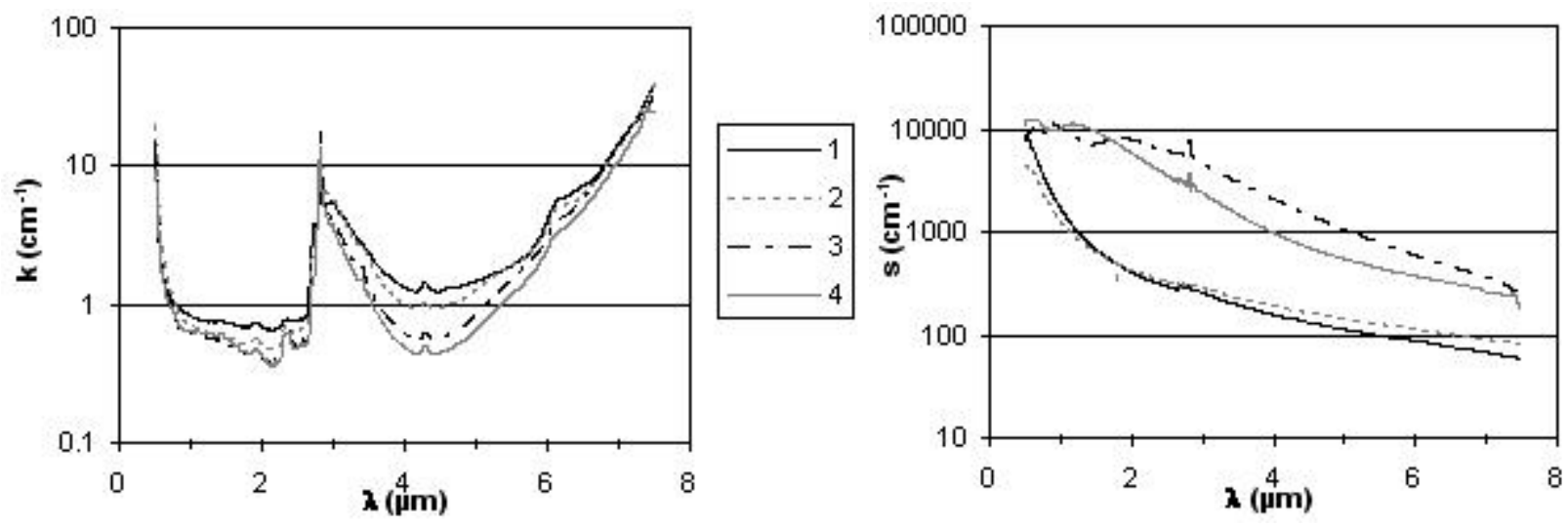

Figure 6. Radiative coefficient spectra of the four coatings annealed at $500^{\circ} \mathrm{C}$ for 24 hours.

These two distinct radiative behaviours can be related to the features of the scatterers, i. e. the pores, and in particular to their mean diameter $d$ in relation to the wavelength within the host material by introducing the complex size parameter $\mathrm{x}=\pi \mathrm{d} /\left(\lambda / \mathrm{m}_{\mathrm{YSZ}}\right)$ ( $\lambda$ being the wavelength in vacuum and $m_{Y S Z}$ the complex optical index of YSZ). When $|\mathrm{x}|<1$, the Rayleigh theory of light scattering is valid: the scattered intensity evolves as $|x|^{4}$ and the light scattering is roughly isotropic. In this study, the Rayleigh limit is found between 1-2 $\mu \mathrm{m}$ for samples with submicron-sized pores (i.e. coatings 1 and 2) whereas it shifts to $7-8 \mu \mathrm{m}$ in the presence of micron-sized pores (coatings 3 and 4). This argument could partly explain the two distinct scattering spectra observed on Fig. 6.

In addition, the scattered intensity depends also on the number of scatterers per unit volume. In order to check this point, the scattering coefficients $\mathrm{s}$ have been normalized by the particle mean crosssection $\pi r^{2}$ and by the mean number of pores per unit volume $\mathrm{N}=\mathrm{P} /\left(4 \pi \mathrm{r}^{3} / 3\right)$ (where $\mathrm{P}$ is the porosity and $r$ the particle mean radius - equal to $d / 2$ - measured by MIP). The evolution of this dimensionless quantity versus the size parameter $|\mathrm{x}|$ is plotted for the four coatings in Fig. 7. 


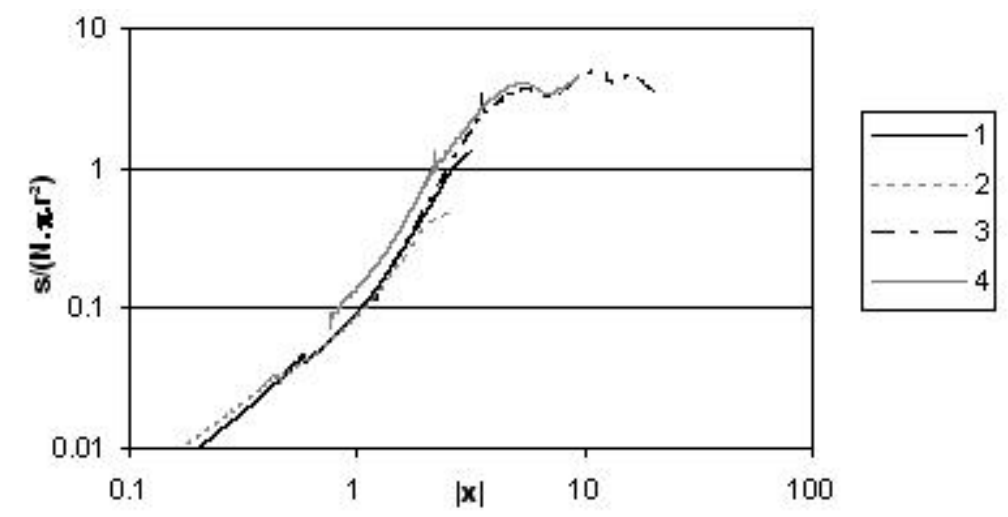

Figure 7. Evolution of the scattering coefficient s normalized by the particle mean cross-section and by the mean number of pores per unit volume versus the size parameter $|\mathrm{x}|$ for the four coatings.

It is noteworthy that the scattering properties of our four studied samples fit into the same pencil of curves. This agreement suggests that the scattering phenomenon within YSZ plasma-sprayed coatings seems to depend mainly on the mean size parameter of the pores and on their volume fraction. However, even if this result is promising, more studies are required to investigate the effects of shape and size distribution of the pores.

\section{TENTATIVE PREDICTION OF THE BULK RADIATIVE PROPERTIES OF THE COATINGS FROM THEIR MICROSTRUCTURAL DESCRIPTIONS}

\section{Framework and assumptions}

A model based on the Mie theory has been implemented in order to predict the radiative properties of the plasma-sprayed coatings. Let us consider a monodisperse population of spherical pores in a homogeneous YSZ host matrix with intrinsic optical properties similar to the ones of the single crystal. The scattering is assumed to be independent so that each pore contribution to the scattered intensity may be summed up. Such an assumption is crucial for simple calculations, but it is very questionable according to the scattering regime map [12] with the size parameter and volume fraction values encountered in this study. A further difficulty arises from the application of the Mie theory in a host medium (here YSZ) which is more absorbing than the scatterers (here the pores). Indeed, the Mie formulation can not be applied here to deduce scattering and absorption coefficients from corresponding cross-sections. To overcome this difficulty, attempts to generalize the Mie theory in an absorbing matrix have been made, but this theorical problem is still discussed nowadays as illustrated in a brief review written by Videen and Sun [13]. Since the definitions of absorption and scattering cross-sections still remain ambiguous, we have retained the formulation suggested by Fardella [14]. This approach allows an evaluation of only the extinction coefficient $\beta$ (sum of the absorption and scattering coefficients) by introducing energy ratios instead of cross-sections.

\section{Modelling results}

Based on the simple microstructure classification reported in Table 2, coatings 1 and 2 should fit at best to the modelling approach. However, a tentative prediction has also been performed for coating 4 although its microstructure description is quite distant from the one of the model. The comparison between model and experiment for coatings 1 and 4 is shown in Fig. 8.

Considering the difficulties involved by the MIE theory and the simplistic microstructure descriptions, a satisfactory agreement between model and experiment is found in both cases since the curve patterns and the orders of magnitude of the extinction coefficients are roughly reproduced by the model.

In particular, the scattering peak below $1.5 \mu \mathrm{m}$ (absorption being negligible over this wavelength range) is properly fit for coating 1 . This result demonstrates the key contribution of submicron-sized 
pores in the scattering process at short wavelengths. Beyond, the modelling leads to an underestimation of the extinction for coating 1, and different arguments can explain this discrepancy: (1) increasing dependence effects with decreasing size parameter, (2) shape effects (spherical shapes are singular within plasma-sprayed samples), (3) possibly non-negligible size distribution effects.

For coating 4, the agreement between model and experiment is rather surprising, especially beyond 3 $\mu \mathrm{m}$. It may emphasize the contribution of the micron-sized pores in the scattering phenomenon in this wavelength range. At shorter wavelengths, the discrepancy is larger and can be explained by the additional scattering contribution of submicron-sized pores detected by MIP. However, these latter results must be balanced since the selected model description does not allow an evaluation of the effects of the lamellar microstructure on the radiative properties of plasma-sprayed coatings.
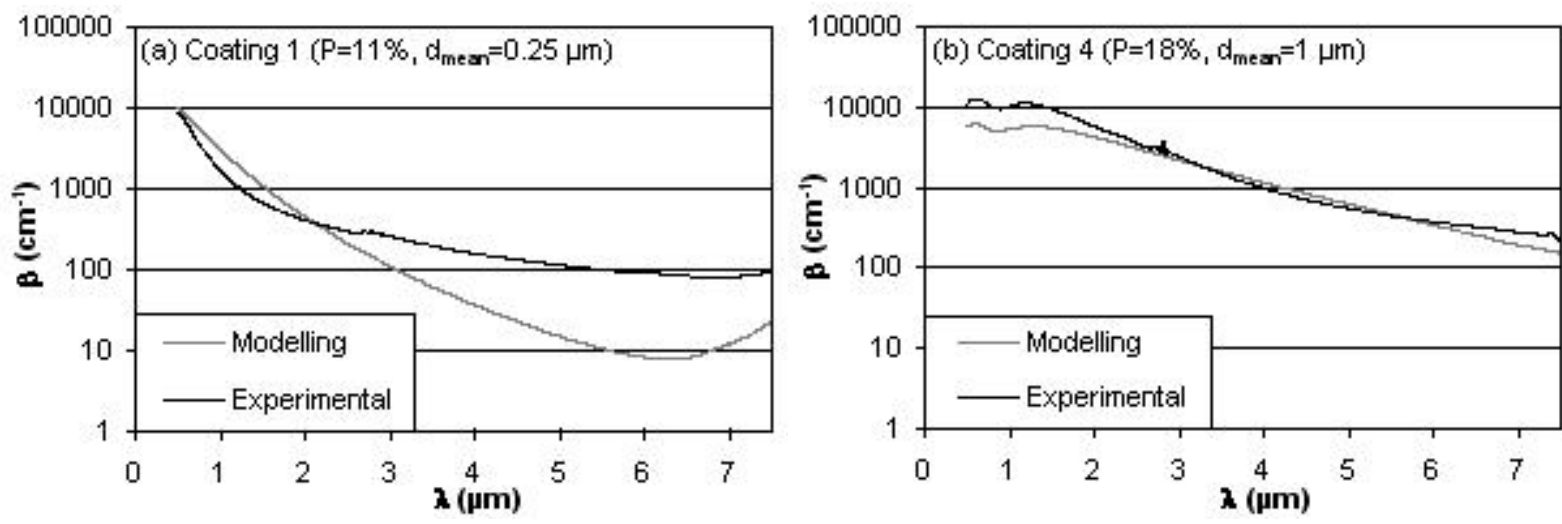

Figure 8. Experimental and simulated extinction coefficient spectra for coatings 1 and 4.

\section{CONCLUSIONS AND PERSPECTIVES}

The optical and radiative properties of four YSZ plasma-sprayed coatings have been characterized over the 0.5-8 $\mu \mathrm{m}$ wavelength range. The as-sprayed samples exhibit differences in both the oxygen under-stoichiometry of the YSZ matrix and the microstructural features. By performing an annealing at $500^{\circ} \mathrm{C}$ in air for 5 minutes to 24 hours, the reflectance and transmittance spectra increase gradually due to the decrase in the matrix absorption. After a 24 hour annealing, no optical variation is observed and the microstructural effects can be investigated. The resulting plasma-sprayed coatings exhibit a high scattering feature that can be related to the volume fraction and the mean size parameter of the pores.

Attempts to predict the radiative properties of the coatings have been carried out by applying the Fardella approach to a monodisperse population of spherical pores embedded in a host absorbing matrix (YSZ). In spite of a simplistic microstructure description and some theoretical difficulties, the agreement between model and experiment is rather satisfactory since the orders of magnitude and the variations versus wavelength of the extinction coefficient are obtained. The modelling indicates that the scattering peak at short wavelengths $(<1.5 \mu \mathrm{m})$ is related to submicron-size pores while the slow decrease in scattering beyond $3 \mu \mathrm{m}$ can be ascribed to micron-sized pores. Further studies with more elaborate models are required to investigate the influences of the shape and the size distribution of the pores and to take into account the dependence effect on the scattering process. Finally, the effect of the lamellar structure on the radiative properties of plasma-sprayed coatings still has to be studied. In this matter, the extension of the four-flux model to a multilayer medium as suggested in [15] could be promising provided that some numerical problems encountered in our first attempts are solved.

\section{REFERENCES}

1. Siegel R. and Spuckler C. M., Analysis of Thermal Radiation Effects on Temperatures in Turbine Engine Thermal Barrier Coatings, Materials Science and Engineering, A245, pp.150-159, 1998. 
2. Eldridge J. I., Spuckler C. M. et al, Infrared Radiative Properties of Yttria-Stabilized Zirconia Thermal Barrier Coatings, Ceramic Engineering Science Proceedings, 23, (4), pp.417-429, 2002.

3. Wahiduzzaman S. and Morel T., Effect of Translucence of Engineering Ceramics on Heat Transfer in Diesel Engines, OAK Ridge Laboratory, Technical Report, ORNL/Sub/88-22042/2, 1992.

4. Ingo G. M., Origin of Darkening in 8wt.\% Yttria-Zirconia Plasma-Sprayed Thermal Barrier Coatings, Journal of the American Ceramic Society, 74, (2), pp.381-386, 1991.

5. Debout V., Vardelle A. et al, Correlation between processing parameters, microstructure and optical properties for plasma-sprayed yttria-stabilized zirconia coatings, 17th International Symposium on Plasma Chemistry, J. Mostaghimi (Ed.), August 7-12, Toronto (Canada), 2005.

6. Moreau C., Gougeon P. et al, On-Line Control of the Plasma Spraying Process by Monitoring the Temperature, Velocity and Trajectory of In-Flight Particles, Proceedings of the 7th National Thermal Spray Conference, June 20-24, Boston (USA), C.C. Berndt, S. Sampath (Ed.), ASM International (Pub.), pp.431-437, 1994.

7. Debout V., Vardelle A. et al, Investigation Of In-Flight Particle Characteristics And Microstructural Effects On Optical Properties of YSZ Plasma-Sprayed Coatings, Proceedings of HTPP 9, St Petersbourg (Russia), Journal of High Temperatures Materials Processing, P. Fauchais and J. Amouroux (Ed.), 2006.

8. Antou G., Montavon G. et al, Exploring Thermal Spray Grey Alumina Coating Pore Network Architecture by Combining Stereological Protocols and Impedance Electrochemical Spectroscopy, Proceedings of the 2006 International Thermal Spray Conference, Seattle (USA), B.R. Marple, M.M. Hyland et al (Ed.), ASM International (Pub.), CD-Rom, 2006.

9. Maheu B., Toulouzian J. N. and Gouesbet G., Four Flux Models to Solve the Scattering Transfer Equation in Terms of Lorentz Mie Parameters, Applied Optics, 23, (19), pp.3353-3362, 1984.

10. Roze C., Girasole T. et al, Average Crossing Parameter and Forward Scattering Ratio Values in Four-Flux Model for Multiple Scattering, Optics Communications, 194, pp.251-263, 2001.

11. Vargas W. E., Amador A. and Niklasson G. A., Diffuse Reflectance of $\mathrm{TiO}_{2}$ Pigmented Paints: Spectral Dependence of the Average Pathlength Parameter and the Forward Scattering Ratio, Optics Communications, 261, pp.71-78, 2006.

12. Tien C. L. and Drolen B. L., Thermal Radiation in Particulate Media with Dependent and Independent Scattering, Annual Review of Numerical Fluid Mechanics and Heat Transfer, Vol. 1, Hemisphere Publishing Corp (Pub.), Washington, 1-32 p., 1987.

13. Videen G. and Sun W., Yet Another Look at Light Scattering from Particles in Absorbing Media, Applied Optics, 42, (33), pp.6724-6727, 2003.

14. Fardella G. and Berthier S., Infrared Emissivity of Inhomogeneous Media, Physica A, 207, pp. 346-351, 1994.

15. Tonon C., Roze C. et al., Four-Flux Model for a Multilayer Plane Absorbing and Scattering Medium: Application to the Optical Degradation of White Paint in a Space Environment, Applied Optics, 40, (22), pp.3718-3725, 2001. 